OPEN ACCESS

Check for updates

\section{E-health StandingTall balance exercise for fall prevention in older people: results of a two year randomised controlled trial}

\author{
Kim Delbaere, ${ }^{1,2}$ Trinidad Valenzuela, ${ }^{3,4}$ Stephen R Lord, ${ }^{1,2}$ Lindy Clemson, ${ }^{3}$ G A Rixt Zijlstra, ${ }^{5}$ \\ Jacqueline C T Close, ${ }^{1,6}$ Thomas Lung, ${ }^{3,7}$ Ashley Woodbury, ${ }^{1}$ Jessica Chow, ${ }^{1}$ Garth McInerney, ${ }^{1}$ \\ Lillian Miles, ${ }^{1}$ Barbara Toson, ${ }^{1,8}$ Nancy Briggs, ${ }^{9}$ Kimberley S van Schooten ${ }^{1,2}$
}

For numbered affiliations see

Correspondence to: K Delbaere, Neuroscience Research Australia, Margarete Ainsworth Building, Barker Street, Randwick,

NSW 2031, Australia

k.delbaere@neura.edu.au (ORCID 0000-0002-5655-0234)

Additional material is published the journal online.

Cite this as: $B M J$ 2021;372:n740 http://dx.doi.org/10.1136/bmj.n740

Accepted: 8 March 2021 end of the article. online only. To view please visit

\section{ABSTRACT}

OBJECTIVE

To test whether StandingTall, a home based, e-health balance exercise programme delivered through an app, could provide an effective, self-managed fall prevention programme for community dwelling older people.

DESIGN

Assessor blinded, randomised controlled trial.

SETTING

Older people living independently in the community in Sydney, Australia.

\section{PARTICIPANTS}

503 people aged 70 years and older who were independent in activities of daily living, without cognitive impairment, progressive neurological disease, or any other unstable or acute medical condition precluding exercise.

\section{INTERVENTIONS}

Participants were block randomised to an intervention group (two hours of StandingTall per week and health education; $n=254$ ) or a control group (health education; $n=249$ ) for two years.

\section{MAIN OUTCOME MEASURES}

The primary outcomes were the rate of falls (number of falls per person year) and the proportion of people who had a fall over 12 months. Secondary outcomes were the number of people who had a fall and the number of injurious falls (resulting in any injury or requiring medical care), adherence, mood, health related quality of life, and activity levels over 24 months; and balance and mobility outcomes over 12 months.

\section{WHAT IS ALREADY KNOWN ON THIS TOPIC}

Balance exercise programmes are among the most effective fall prevention strategies, with fall reduction rates of $23 \%$ in older people who live in the community

Despite strong evidence that falls can be prevented, sustained full adherence in effective trials is poor, with pooled adherence rates of $21 \%$ (range $0-68 \%$ ) at 12 months

Previous studies have shown that e-health technology can deliver unsupervised balance exercises to older people, with good adherence rates

\section{WHAT THIS STUDY ADDS}

This study is a large, long term, and methodologically robust trial examining technology driven exercise as a strategy to prevent falls in older people

Over two years, the StandingTall programme significantly reduced the rate of falls and the rate of injurious falls with dose adherence rates of $30-40 \%$

\section{RESULTS}

The fall rates were not statistically different in the two groups after the first 12 months ( 0.60 falls per year (standard deviation 1.05) in the intervention group; $0.76(1.25)$ in the control group; incidence rate ratio $0.82,95 \%$ confidence interval 0.66 to $1.02, \mathrm{P}=0.070$ ). Additionally, the proportion of people who fell was not statistically different at 12 months $34.6 \%$ in intervention group, $40.2 \%$ in control group; relative risk $0.90,95 \%$ confidence interval 0.72 to 1.12 , $\mathrm{P}=0.348$ ). However, the intervention group had a $16 \%$ lower rate of falls over 24 months (incidence rate ratio $0.84,95 \%$ confidence interval 0.72 to $0.98, P=0.027$ ) and a $20 \%$ lower rate of injurious falls over 24 months compared with the control group (incidence rate ratio $0.80,95 \%$ confidence interval 0.66 to 0.98 , $\mathrm{P}=0.031$ ). Both groups had a similar proportion of people who fell over 24 months (relative risk 0.87 , $95 \%$ confidence interval 0.74 to $1.02, \mathrm{P}=0.077$ ). In the intervention group, $68.1 \%$ and $52.0 \%$ of participants exercised for a median of $114.0 \mathrm{~min} /$ week (interquartile range 53.5) after 12 months and 120.4 min/week (38.6) after 24 months, respectively. Groups remained similar in mood and activity levels. The intervention group had a 0.03 (95\% confidence interval 0.01 to 0.06 ) improvement on the EQ-5D-5L (EuroQol five dimension five level) utility score at six months, and an improvement in standing balance of $11 \mathrm{~s}(95 \%$ confidence interval 2 to $19 \mathrm{~s})$ at six months and $10 \mathrm{~s}$ (1 to $19 \mathrm{~s})$ at 12 months. No serious training related adverse events occurred.

\section{CONCLUSIONS}

The StandingTall balance exercise programme did not significantly affect the primary outcomes of this study. However, the programme significantly reduced the rate of falls and injurious falls over two years, with similar but not statistically significant effects at 12 months. E-health exercise programmes could provide promising scalable fall prevention strategies.

\section{TRIAL REGISTRATION}

ACTRN12615000138583

\section{Introduction}

Falls and fall related injuries have persisted over the past three decades as a leading cause of morbidity and mortality in older people. ${ }^{1}$ With a rapidly ageing population globally, sustainable access to evidence based, cost effective fall prevention programmes is a priority. Evidence from high quality systematic reviews and meta-regressions has confirmed that well designed exercise programmes are among the most effective fall prevention strategies for community dwelling 
older people, with fall reduction rates averaging $23 \% .^{2}$ However, to achieve similar effectiveness at a population level, we need a programme that people can access easily and adhere to in the long term. Previous studies have found that older people prefer home based exercises and that the inclusion of balance exercises is associated with higher adherence. ${ }^{3}$ Nevertheless, sustained adherence to prescribed home exercise programmes is low, with pooled estimates of $21 \%$ (range 0-68\%). ${ }^{4}$ Studies providing a physiotherapist led programme or a moderate level of home visits (that is, less than one home visit per month and more than two home visits in total) achieve higher levels of adherence $^{4}$; however, such measures substantially increase the cost and reduce the feasibility as a population approach.

Digital technology can provide engaging and widely accessible methods for delivery of exercise programmes to enhance long term motivation and adherence at relatively low cost. $^{5}$ However, the provision of a well designed, unsupervised exercise programme that is tailored and progressive in nature, yet safe, could be a challenge. StandingTall is a home based, e-health balance exercise programme provided through an app that was developed by using principles of consumer design to ensure an appropriate and user friendly interface for older people. Behavioural change strategies are incorporated to enhance exercise uptake and long term adherence. ${ }^{6}$

This randomised controlled trial aimed to determine the effect of StandingTall on the recommended set of core outcomes for fall prevention trials in older people (fall rate, number of people who fall and those who have an injurious fall; and known fall risk factors, including balance, gait, concern about falling, health related quality of life, and physical activity levels). ${ }^{7}$ The trial had a 24 month follow-up period and compared the outcomes of the intervention with a health promotion education control programme. We hypothesised that StandingTall would lead to a reduced fall rate compared with a control group with minimal intervention.

\section{Methods}

\section{Study design}

We conducted a prospective, assessor blinded, two arm, parallel randomised controlled trial with two year follow-up in Sydney, Australia. The trial was approved by the University of New South Wales ethics committee in December 2014 (HC\#14/266) and was registered prospectively in the Australian and New Zealand Clinical Trials Registry (ACTRN12615000138583) on 13 February 2015. The study protocol was published in $2015 .^{8}$ The statistical analysis plan was preregistered in October 2018 through the OpenScience framework (https://osf.io/42gje/) before completion of data collection in November 2019. We used the CONSORT (consolidated standards of reporting trials) statement, ICMJE recommendations, and TiDieR (template for intervention description and replication) checklist when preparing this article.

\section{Participants}

We recruited community dwelling older people in the Sydney metropolitan area by using flyers, printed advertisements in local newspapers, presentations at residential and community senior centres, and word of mouth. Study participants lived on average $12 \mathrm{~km}$ (range 1.2-46.9 km) from Neuroscience Research Australia (Randwick, NSW). After initial screening by telephone, eligible people were invited to participate if they were aged 70 years or older, living in the community, independent in activities of daily living, able to walk household distances without the use of a walking aid, and willing and able to give informed consent and comply with the study protocol. People were excluded if they had an unstable or acute medical condition that precluded exercise participation, suffered from a progressive neurological condition (such as Parkinson's disease or multiple sclerosis), were cognitively impaired as defined by a Pfeiffer short portable mental status questionnaire score less than $8,{ }^{9}$ or were currently participating in a fall prevention programme. Eligibility was determined after informed verbal consent. People who were eligible and agreed to participate in the study were asked to provide informed written consent.

\section{Randomisation and masking}

Participants were randomised after completion of the baseline assessment. Permuted block randomisation with mixed block lengths of four and six was applied to form two groups of similar size (allocation ratio 1:1). People living in the same household were treated as one unit to avoid contamination. Allocation was performed centrally using a custom randomisation programme by an investigator not involved in participant assessments or delivery of the intervention. Allocation concealment was ensured because the randomisation code was only released after the baseline assessment was completed. Only the first 226 participants were invited for repeated physical tests to reduce costs and participants' time. Outcome assessors were blinded to study group assignment throughout the trial. Statistical analyses were performed blinded for intervention or control group allocation.

\section{Procedures}

All participants received a tablet computer with a health promotion education programme that focused on health related information relevant to older people, in addition to usual care, for two years. This health promotion education programme comprised weekly fact sheets (104 in total) with information on healthy diet, drugs, fall risk factors, and exercise. Tablet based health education alone was chosen as the active control intervention to regulate the use of technology and allow data collection (such as number of falls during the trial period) through a tablet computer for both groups. Participants received a manual on how to use the tablet computer. After their baseline assessment, participants received guidance on the basic features of the tablet computer and health promotion education programme. 
The intervention group received the StandingTall programme, with exercise equipment (foam cushion, stepping box, exercise mat), in addition to the health promotion education programme and usual care that was received by the control group. The StandingTall intervention consisted of balance exercises delivered through a tablet computer in the participants' homes with embedded behavioural change techniques, including a weekly calendar for scheduling exercises, goal setting, and educational fact sheets. The exercises focus on standing balance, targeted stepping, and step-up (box) exercises. More information about the programme can be found in the study protocol ${ }^{8}$ and online (https://www.standingtall. org.au/). Participants were asked to exercise for at least two hours per week for the duration of the trial, in line with the international recommendations for fall prevention at the time of the study. ${ }^{2}$ The intervention was introduced gradually; participants started with $40 \mathrm{~min} /$ week of exercise, which was increased by 20 min fortnightly until participants reached the required amount of two hours per week in week 9.

StandingTall delivers individually tailored balance exercises that increase in difficulty over time; the programme also allows people to choose the time and duration of their exercise sessions. The intensity of the balance exercises is monitored by using a selfreport modified rate of perceived exertion scale and is adjusted as performance changes throughout the trial without the need for supervision. Exercise adherence (volume and frequency) was monitored for two years after automatic data transfer to a server. During the first six months, participants were encouraged to inform the research team when they were going away or would not be able to exercise for a few weeks. Participants who did not inform the team and did not reach $100 \mathrm{~min} /$ week for two consecutive weeks were contacted by telephone so that the reason for nonadherence could be recorded, any issues related to the programme could be discussed, and the team could encourage adherence. These calls stopped after six months to gain a better understanding of behavioural change and long term exercise adherence.

Intervention group participants received two home visits. During the first visit, a qualified exercise physiologist instructed the participant on how to use the StandingTall programme; this visit occurred between one and three weeks after the baseline assessment and lasted approximately one hour. The second home visit after one month lasted approximately $30 \mathrm{~min}$ and ensured safe use of the programme and progression of training. Control group participants received two phone calls by qualified exercise physiologists at the same time points to discuss any issues with accessing the health education programme and using additional features of the tablet computer.

\section{Outcomes}

The primary outcome measures were the rate of falls and the proportion of people who fell over the first 12 months of the trial. A fall was defined as 'an unexpected event in which the participant comes to rest on the ground, floor or lower level'. ${ }^{7}$ Falls were monitored by using prospective weekly fall diaries through the tablet computer (completed from baseline assessment for 24 months). Fall information was automatically uploaded to a database. Research staff contacted participants by telephone at the end of each month when their fall diaries were incomplete to record missing data. The falls database was checked, reviewed, and locked before group allocation was unmasked. Falls that occurred up to one year after randomisation were included in the primary analysis. Falls that occurred up to two years after randomisation were included as secondary fall outcomes. Injurious falls were defined as falls that resulted in any injury (eg, bruises, cuts or grazes, joint dislocations, sprains or strains, fractures, pain), or falls that required medical care (eg, visit to physician or emergency department).

Secondary outcome measures were assessed at baseline, at six months to examine acute effects, and at 12,18 , and 24 months to examine retention effects. These measures included common fall risk factors: laboratory based balance and neuropsychological assessments (at baseline and at six and 12 months after baseline assessment in the first 226 participants), and remote measures (taken at home) of wellbeing, quality of life, and activity levels (at baseline and at six, 12, 18, and 24 months after baseline assessment in all participants). Physiological fall risk was assessed using the physiological profile assessment. ${ }^{10}$ Balance, functional mobility, and gait were evaluated by using tests of standing balance (standing with feet in different positions for a maximum of $30 \mathrm{~s}$ per condition: feet together, near tandem, and tandem on floor and foam cushion, and left and right foot on floor; sum of durations for all eight conditions), maximum forwardbackwards and controlled leaning balance, ${ }^{10} 11$ timed sit-to-stand ${ }^{12}$ and up-and-go tests, ${ }^{13}$ short physical performance battery, ${ }^{14}$ and self-selected walking speed over $10 \mathrm{~m} .{ }^{15}$ Stepping performance was assessed with choice, Stroop and inhibitory stepping reaction time tests. ${ }^{16} 17$ Cognitive function was measured with the Montreal cognitive assessment ${ }^{18}$ for global cognition, trail making tests ${ }^{19}$ for set shifting, and the Victoria Stroop task ${ }^{20}$ for response inhibition. Psychological outcome measures were assessed by using the iconographical falls efficacy scale (concern about falling), ${ }^{21}$ the nine item patient health questionnaire $(\operatorname{mood})^{22}$ and the COMPAS-W scale (wellbeing). ${ }^{23}$ Health related quality of life was measured with the 12 item WHO disability assessment schedule, ${ }^{24}$ the EuroQol five dimension five level (EQ-5D-5L) questionnaire, ${ }^{25}$ and the AQOL-6D (20 item assessment of quality of life six dimensions) questionnaire. ${ }^{26}$

Detailed self-report information on frequency and duration of physical activity was evaluated with the incidental and planned exercise questionnaire. ${ }^{27}$ Daily life activity was assessed with the McRoberts MoveMonitor (McRoberts, Netherlands) as the average duration of daily walking and standing, and the number of walking and standing bouts per $\operatorname{day}^{28}$; a 
bout was defined as a period of consecutive activity. Because participants were instructed to remove the device before going to bed, we required a minimum wear duration of 12 hours per day on one or more days for daily life activity data to be included in the analysis. Daily life activity data were collected over a median of six days (interquartile range one day) for both groups.

Process outcome measures included exercise duration and were captured through the tablet computer. Because participants were allowed exercise breaks when they were sick or went on holiday, we averaged weekly exercise duration as median values for participants as a robust measure of central tendency. We obtained subjective user experience by assessing usability, enjoyment, and exercise self-efficacy with the system usability scale, ${ }^{29}$ the physical activity enjoyment scale $^{30}$ the exercise self-efficacy scale, ${ }^{31}$ and the attitudes to falls related interventions scale. ${ }^{32}$

All outcome measures were assessed by trained exercise physiologists or physiotherapists who were blinded to group allocation. We assessed safety in terms of adverse events, which were defined as any fall related to the prescribed exercise programme or involving the intervention equipment.

\section{Statistical analysis}

Sample size calculation-based on previous evidence, we carried out an a priori sample size calculation ${ }^{8}$ in Stata using a custom code with 5000 simulations. The calculation showed that 500 participants were required to achieve $80 \%$ power to find a fall rate reduction of $33 \%$ (incidence rate ratio of 0.67 ) in the intervention versus the control group that is statistically significant at a $\mathrm{P}$ value less than 0.05 (considering an overdispersion of 1.2, 0.8 falls/person year in the control group, and a follow-up duration of 22 months to account for $20 \%$ dropout rate). We then ran power calculations in $\mathrm{G}^{\star}$ Power (version 3.1.7) for our secondary outcomes (considering an analysis of variance design with four measurements and 20\% dropout rate). These calculations showed that we would have $90 \%$ power to detect a statistically significant $(\mathrm{P}<0.05)$ small reduction (effect size $\mathrm{f}=0.15$ ) in concern about falling in the intervention group versus the control group, assuming a within subject correlation of $0.75 .^{8}$ A subsample of 200 participants with repeat physical assessments would provide us with 95\% power to detect a statistically significant $(\mathrm{P}<0.05)$ large reduction (effect size $\mathrm{f}=0.38$ ) in postural sway in the intervention group versus the control group, assuming a within subject correlation of $0.76 .^{8}$

Analysis plan-analyses were conducted according to the predefined statistical analysis plan, as registered on the OpenScience framework (https://osf.io/42gje/). We coded data to maintain group allocation blinding during analysis. Effectiveness analyses of the primary outcome were conducted on an intention-to-treat basis by a statistician (BT or NB) and independently replicated by one of the investigators (KSvS). The $\alpha$ level was set to $5 \%$. Analyses were performed with Stata (version 16, Stata Corp) and SPSS (version 25, IBM Corp).
Missing data-participants who were randomly assigned to a group were included in the analysis irrespective of their level of compliance with their group assignment, which was in line with intention-to-treat principles. The primary outcome measures (number of falls per person year and proportion of people who fell over 12 months) were analysed without imputation or adjustment for descriptive characteristics, and with correction for follow-up duration when appropriate. We assumed that the faller status of people with incomplete follow-up ( $n=66$ at 12 months and $n=188$ at 24 months, distributed evenly over the two groups) was maintained during censoring. We used Little's missing completely at random test to determine the missing data patterns of secondary outcome measures. The secondary outcome measures were imputed using estimated means single imputation if they were missing completely at random; or under the assumption of missing at random using multiple imputation to create 20 imputation datasets under joint multivariate normal imputation $^{33}$ if they were not missing completely at random. Psychological wellbeing, health related quality of life, and physical activity questionnaire data were missing for 58 out of a total of 503 people at six months, for 82 people at 12 months, for 98 people at 18 months, and for 99 people at 24 months. Daily life activity monitoring data were unavailable for 21 people at baseline, 101 people at six months, 138 people at 12 months, 148 people at 18 months, and 156 people at 24 months. Clinic based balance and neuropsychological assessment data were missing for 42 people at six months and for 47 people at 12 months. These data were missing because of dropout, scheduling issues, non-adherence, or technical problems. Little's missing completely at random test indicated that all data were missing at random with respect to participant baseline characteristics.

Primary outcomes-primary outcomes were the number of falls per person year, and the proportion of people who fell over 12 months. The number of falls per person year was analysed using Poisson regression to estimate the difference in fall rates between the two groups. Incidence rate ratios and 95\% confidence intervals are reported. Poisson regression was selected over negative binomial regression (as a priori registered in our statistical analysis plan, but not in our protocol paper) to allow for a direct comparison with our planned complier average causal effects analysis because this analysis was based on a Poisson model. Online appendix 1 presents the results of the negative binomial regression. Days of follow-up was included as an exposure term in these models; that is, the natural logarithm of the days of follow-up was added as an offset. We examined the proportion of people who fell in the two groups by using modified Poisson regression models for binary outcomes. Faller status was compared (no falls $v$ at least one fall) and relative risks and 95\% confidence intervals are reported.

Secondary outcomes-secondary fall outcomes were the number of falls, the complier averaged causal effect, the number of injurious falls, the proportion of 
people who fell, and the proportion of people who had injurious falls at 24 months. We used instrumental variable regression to correct for imperfect participant adherence and to gain insight into efficacy by estimating the complier averaged causal effect. We used a 2000 times bootstrapped, two stage complier averaged causal effect estimator composed of a linear regression with adherence as the dependent variable and group as the independent variable to obtain an estimate for adherence. A robust Poisson regression was then performed, with falls as the dependent variable and the natural logarithm of follow-up in days as exposure to estimate the effect of the intervention among people with perfect adherence. The number of injurious falls per person year was analysed using Poisson regression to estimate the difference in injurious fall rates between the two groups. We analysed secondary non-fall outcome measures with robust generalised linear models using an exchangeable working correlation matrix and compared the change in scores over time at six, 12 , 18, and 24 months between the two groups. When the residuals of the generalised linear models deviated from normality, we used a 1000 times bootstrap for each imputation dataset to obtain confidence intervals.

\section{Patient and public involvement}

StandingTall was developed using consumer design principles. A group of older people were involved during the development of the StandingTall application. They were asked to evaluate an early version on its usability and age appropriateness as a means to engage in fall prevention exercises using tablet based technology. A two week feasibility study was conducted in 10 community dwelling older people in November 2013. The average age of the participants was 77.5 years (range 67-82 years), and six participants were women. Physiological profile assessment scores ranged from mild to marked (median z score 1.68; range 0.79-2.94) and seven participants had experienced falls in the previous year. Adherence was high, with participants reporting that the programme was suitable for older people. There was no other formal patient and public involvement in this study.

\section{Results}

Between February 2015 and October 2017, 823 people were screened (fig 1). Of these, 503 people were included in the study and randomly assigned to the intervention group $(\mathrm{n}=254)$ or the control group $(n=249)$. We lost 90 participants during the two year follow-up (intervention group, $\mathrm{n}=53$; control group, $\mathrm{n}=37$ ) and 46 participants in the intervention group discontinued the intervention but continued to contribute data. Table 1 presents baseline characteristics of all participants.

\section{Effect on primary fall outcomes}

Rate of falls at 12 months-the incidence rate of falls over the first 12 months was 0.61 (95\% confidence interval 0.52 to 0.71 ) in the intervention group and 0.75 (0.64 to 0.85$)$ in the control group. The difference in fall rate was not statistically different, with an incidence rate ratio of 0.82 (95\% confidence interval 0.66 to $1.02, \mathrm{P}=0.070$ ) in the intervention group compared with the control group (see fig 2).

Proportion of people who fell over first 12 monthsoverall, 188 participants $(37.4 \%$ in total; $34.6 \%$ in the intervention group and $40.2 \%$ in the control group) fell at least once in the 12 month follow-up period. Participants in both groups were equally likely to fall at least once, with a relative risk of 0.90 (95\% confidence interval 0.72 to $1.12, \mathrm{P}=0.35$ ) in the intervention group compared with the control group.

\section{Effect on secondary fall outcomes}

Rate of falls at 24 months-the incidence rate of falls over the 24 month follow-up was 1.17 (95\% confidence interval 1.03 to 1.30 ) in the intervention group and 1.39 (1.25 to 1.53 ) in the control group. The difference in fall rate was statistically different, with an incidence rate ratio of 0.84 ( $95 \%$ confidence interval 0.72 to $0.98, \mathrm{P}=0.03)$ in the intervention group compared with the control group.

Rate of falls through complier averaged causal effects at 24 months-complier averaged causal effect analysis revealed an incidence rate ratio of 0.67 (95\% confidence interval 0.21 to $1.13, \mathrm{P}=0.22$ ) in the intervention group compared with the control group. This figure was similar to that reported in the intentionto-treat analysis.

Rate of injurious falls at 24 months-the incidence rate of injurious falls over the 24 month follow-up was 0.71 (95\% confidence interval 0.60 to 0.81 ) in the intervention group and 0.88 (0.76 to 0.99 ) in the control group. The difference in injurious fall rate was statistically different, with an incidence rate ratio of 0.80 ( 0.66 to $0.98, \mathrm{P}=0.03)$ in the intervention group compared with the control group.

Proportion of people who fell at 24 months -270 participants (53.7\%) fell at least once in the 24 month follow-up. Participants in both groups were equally likely to fall at least once, with a relative risk of 0.87 (95\% confidence interval 0.74 to $1.02, \mathrm{P}=0.077$ ) in the intervention group compared with the control group.

Proportion of people who had injurious falls at 24 months-210 participants $(41.7 \%$ in total; $37.4 \%$ in the intervention group and $46.2 \%$ in the control group) experienced an injurious fall during the 24 month follow-up. Participants in both groups were equally likely to have injurious falls, with a relative risk of 0.87 (95\% confidence interval 0.71 to $1.06, \mathrm{P}=0.17$ ).

Effect on secondary outcomes of wellbeing, quality of life, and activity levels

We found no significant difference in psychological wellbeing or physical activity levels at six, 12, 18, and 24 months in the two groups (table 2). We found a small improvement of 0.03 (95\% confidence interval 0.01 to 0.06) on the EQ-5D-5L utility score at six months in the intervention group compared with the control group. All other health related quality of life measures showed no difference between the two groups at all time points. 


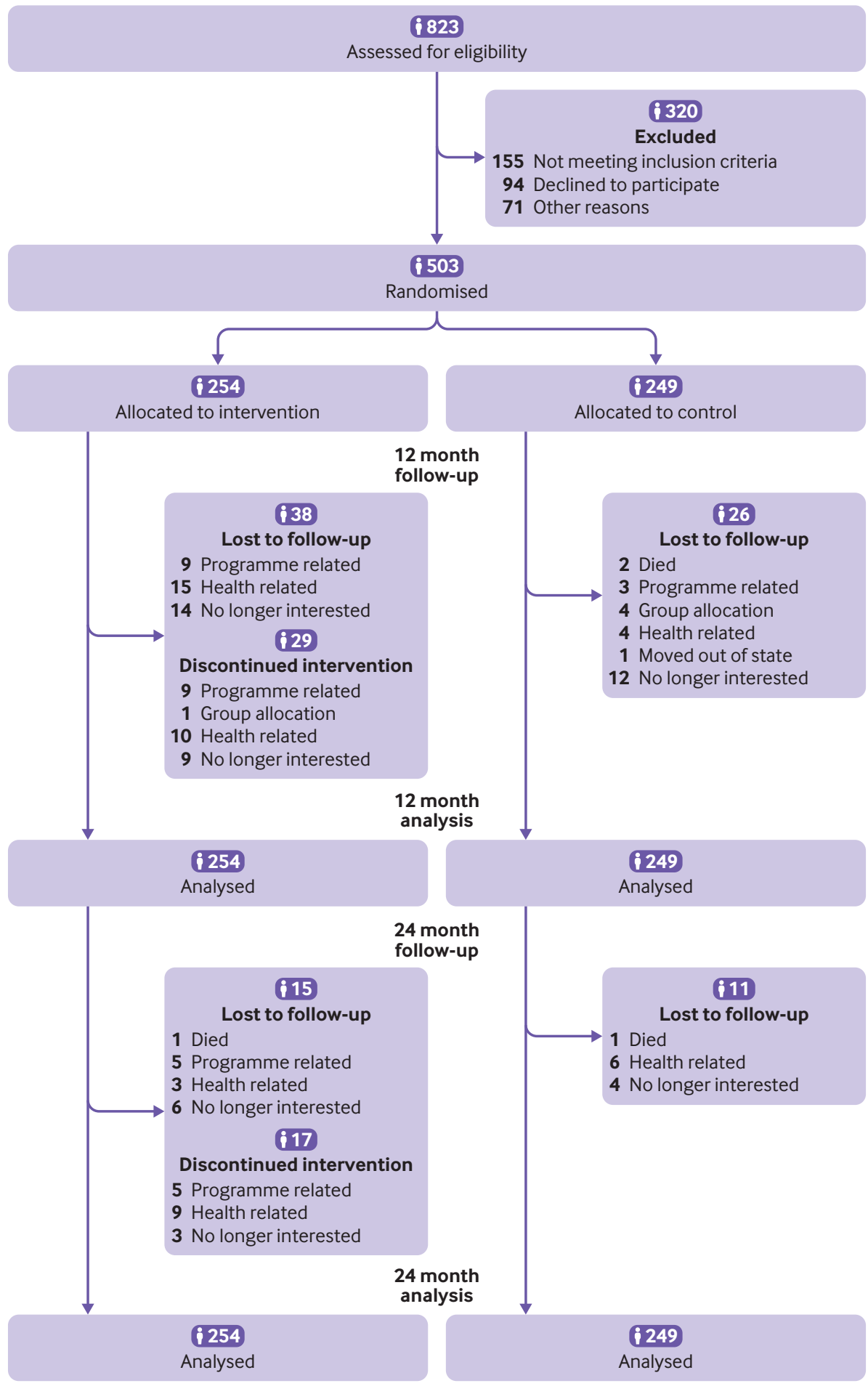

Fig 1 | Flowchart of study recruitment and retention

Effect on secondary outcomes of balance and neuropsychological assessments

The 226 participants ( $45 \%$ of the total sample; 114 in intervention group and 112 in control group) who were invited for laboratory reassessments were on average 1.1 years older (78.0 (standard deviation 5.4 ) years $v 76.9$ (5.5) years in those not invited; $\mathrm{t}(501)=-2.29$, $\mathrm{P}=0.02$ ) and scored 0.40 points higher on physiological fall risk (measured with physiological profile assessment: 1.10 (standard deviation 0.82) $v 0.70$ (0.90) in those not invited; $\mathrm{t}(501)=-5.06, \mathrm{P}<0.001)$. No other important differences in baseline characteristics were found between these groups. We observed a significant improvement in standing balance at six and 12 months (11 s, 95\% confidence interval 3 to 19 s, and $10 \mathrm{~s}, 1$ to $19 \mathrm{~s}$, respectively) in the intervention group compared with the control group (table 3 ). We found no significant difference in physiological fall risk, 


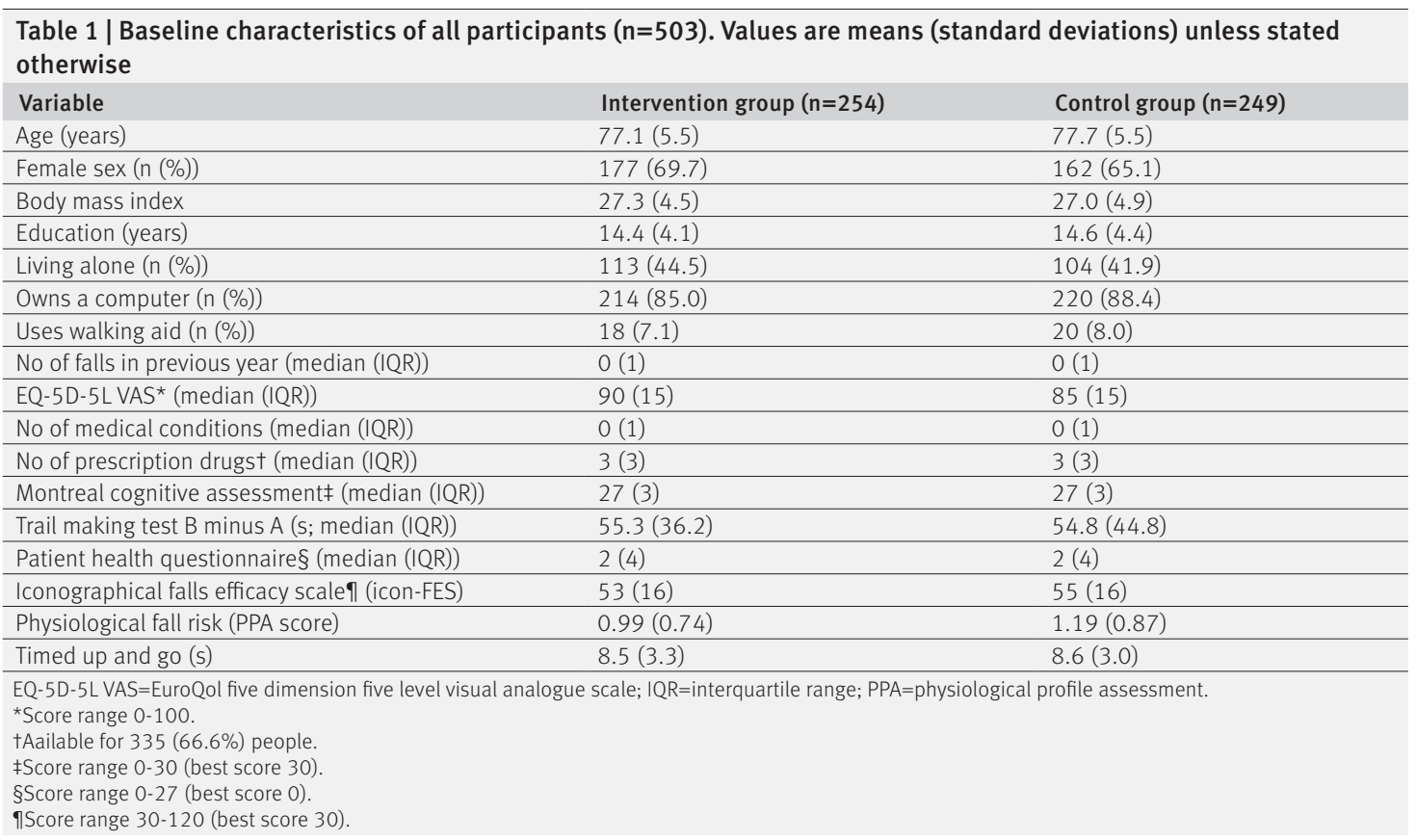

maximum forward-backwards and controlled leaning balance, functional mobility and gait tests, stepping performance, or cognitive and executive functions at six or 12 months between the two groups.

\section{Subgroup analyses}

Planned subgroup analyses in participants who did or did not experience falls in the past 12 months or had low or high physiological fall risk, concern about falling, or executive function scores (median splits on physiological profile assessment, iconographical falls efficacy scale, and trail making test B) at baseline suggested no mediation on rate of falls (all $\mathrm{P}=0.058$; see online appendix 2). The subgroup analysis suggested mediation by baseline status of physiological fall risk on physiological fall risk and by baseline status of concern about falling on concern about falling $(\mathrm{P}=0.004$ and $\mathrm{P}=0.027$, respectively; see online appendix 3). People with lower physiological fall risk at baseline had a significantly greater improvement in physiological fall risk at six months of 0.52 points ( $95 \%$ confidence interval 0.17 to 0.88 points). People with higher concern about falling at baseline had a significantly lower improvement in concern about falling at 12 months of -5 points ( -9 to -1 points).

\section{Process outcomes}

Adverse events-five falls occurred in three participants from the intervention group while exercising, which led to minor injuries (grazes, bruising, cuts). These falls were directly related to the intervention. Three falls occurred during exercise sessions and two were caused by tripping over exercise equipment.

Adherence-in the intervention group, a total of 51 participants $(20.1 \%)$ had a median adherence of 0 min/week at six months, 81 (31.9\%) at 12 months, 104 (40.9\%) at 18 months, and $122(48.0 \%)$ at 24 months, either because of dropout or non-usage attrition (see fig 1). The remaining participants exercised for a median of $105.0 \mathrm{~min} /$ week (interquartile range 58.5, $\mathrm{n}=203$ ) over the first six months, $114.0 \mathrm{~min} /$ week (53.5, $\mathrm{n}=173$ ) over the first 12 months, $120.0 \mathrm{~min} /$ week (39.3, $\mathrm{n}=150)$ over 18 months, and $120.3 \mathrm{~min} /$ week (38.6, $n=132$ ) over the full 24 months. Overall,

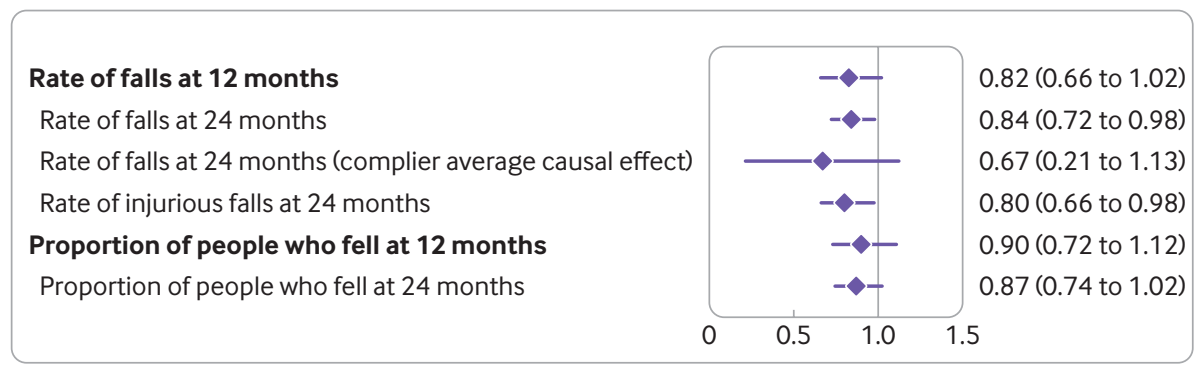

Fig 2 | Effect of StandingTall, a home based, e-health balance exercise programme, on rate of falls, rate of injurious falls, and proportion of people who fell at 12 months and 24 months. Values are incidence rate ratio (rate of falls) or relative risk (proportion of people who fell) with corresponding $95 \%$ confidence interval. Vertical line indicates no difference between groups (incidence rate ratio or relative risk=1). Primary outcome measures are given in bold 
$40.0 \%, 34.1 \%, 33.1 \%$, and $29.8 \%$ of participants in the intervention group achieved the prescribed dose over six, 12, 18, and 24 months, respectively.

Attitudes and usability-attitudes to falls related interventions and exercise self-efficacy scale scores at baseline were similar for both groups $(\mathrm{P}=0.595$ and $\mathrm{P}=0.681$, respectively), with medians of 42 (interquartile range 9) and 86 (22) in the control group versus 42 (8) and 87 (23) in the intervention group. We repeated both measures and also obtained system usability scale and physical activity enjoyment scale scores for the intervention group at six, 12, 18, and 24 months. Attitudes to falls related interventions score declined over time $(\mathrm{P}<0.001)$ from a median of 42 (interquartile range 8) at baseline, to 40 (10) at six months, 39 (11) at 12 months, 39 (14) at 18 months, and 35 (18) at 24 months; these results suggest reduced intentions to continue the intervention. Exercise self-efficacy scale score also declined over time $(\mathrm{P}<0.001)$ from a median of 87 (interquartile range 23) at baseline, to 75 (28) at six months, 70 (27) at 12 months, 69 (39) at 18 months, and 59 (41) at 24 months; these results suggest reduced exercise self-efficacy. Physical activity enjoyment scale and system usability scale scores remained stable over time ( $\mathrm{P}=0.36$ and $\mathrm{P}=0.70$, respectively), with medians of 27 (interquartile range 14) and 4.4 (0.8), respectively.

\section{Discussion}

\section{Principal findings}

We observed no significant effects on our primary outcomes-rate of falls and proportion of people who fell-at 12 months. However, at 24 months we did observe a significant reduction in fall rate (16\%)

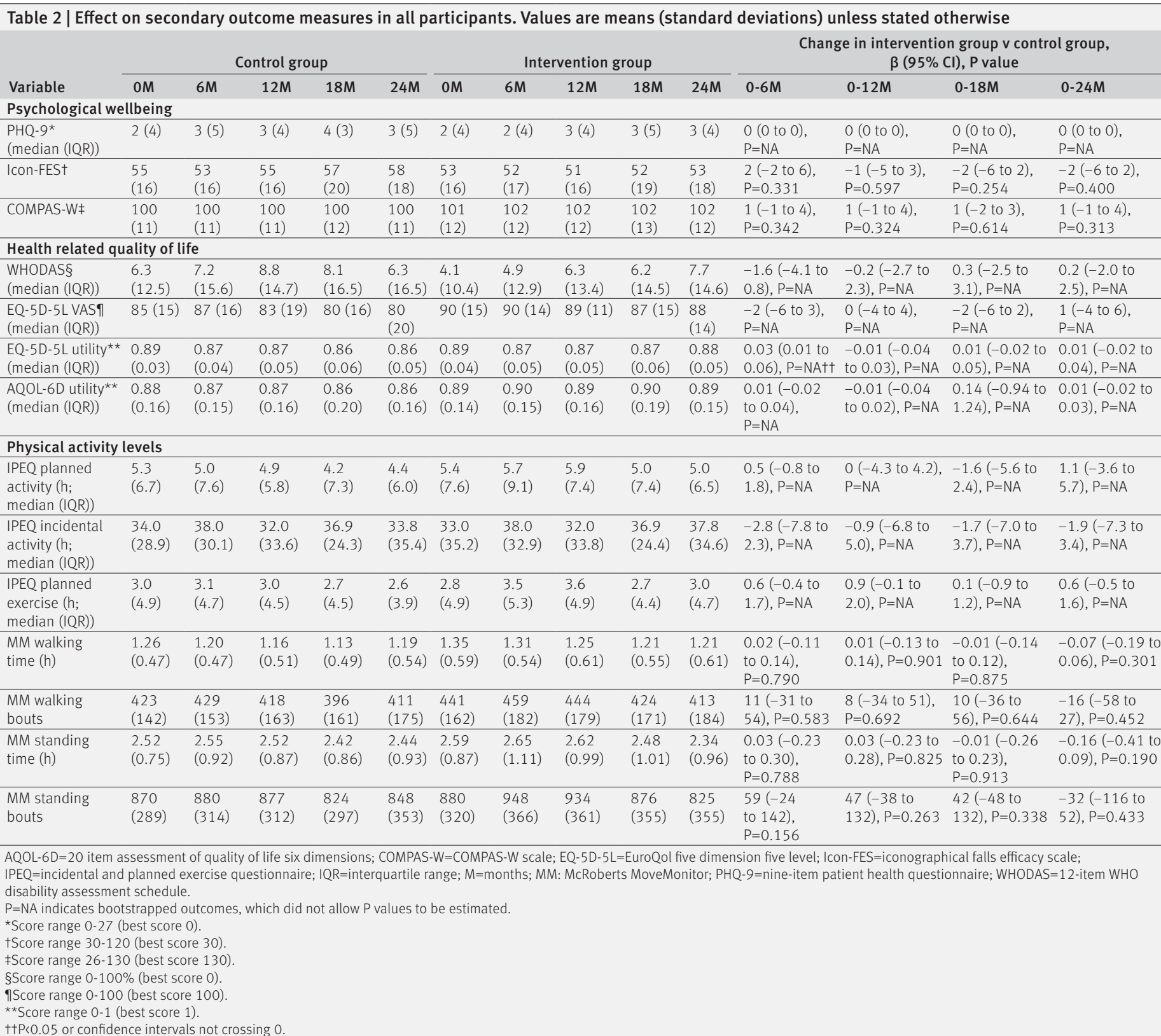


and in injurious fall rate $(20 \%)$. The effect size of an $18 \%$ reduction in fall rate at 12 months was similar to that at 24 months, although it was not statistically significant $(\mathrm{P}=0.07)$. The observed reduction in fall rate is comparable to that reported in previous studies $-21 \%$ reduction with individually delivered exercise programmes over 12 months and $14 \%$ over 24 months. ${ }^{234}$ However, the $20 \%$ reduction in the rate of injurious falls over 24 months seems to be higher than the $12 \%$ reduction previously reported in other studies. ${ }^{35}$ These findings indicate that technology can be used to deliver an e-health balance exercise programme to older people that is effective at reducing fall rates and injurious fall rates over 24 months.

Secondary outcome analyses were not able to clearly highlight the pathway through which the reduction in rate of falls and injurious falls was achieved. While a number of research studies testing the effects of exercise interventions on balance in older people have shown an effect, systematic review findings have suggested that the evidence for a moderate effect is weak. ${ }^{36}$ In a subgroup of 226 participants, we observed a major improvement in standing balance at six and 12 months; however, this observation was not confirmed through other balance and functional mobility measures. We did not repeat these assess- ments at 24 months when a significant reduction in falls was observed. The StandingTall programme includes a monthly balance assessment that consists of maintaining a standing posture with the feet in different positions; it is possible that the repeated practice carried over to laboratory assessments for participants in the intervention group. This trial might have been underpowered for detecting differences in fall risk factors because our sample had a lower fall risk than anticipated. The a priori sample size calculation was based on a sample with a mean physiological fall risk score (physiological profile assessment) of 1.9 (standard deviation 1.1), which is a full point higher than that of the current sample (0.88, standard deviation 0.88). ${ }^{8}$ Interestingly, our preregistered subgroup analyses found no significant modification of fall rates, but did suggest significant modification of the assessment outcomes at 12 months, with people with lower physiological fall risk and lower concern about falling benefitting more. Further research is required to confirm the effectiveness of StandingTall in older people with an increased risk of falling. Quality of life measured with the EQ-5D-5L utility index also showed a small, but potentially clinically relevant ${ }^{37}$ improvement at six months, however no significant differences were found at 12 or 24 months.

\begin{tabular}{|c|c|c|c|c|c|c|c|c|}
\hline \multirow[b]{2}{*}{ Variable } & \multicolumn{3}{|c|}{ Control group } & \multicolumn{3}{|c|}{ Intervention group } & \multicolumn{2}{|c|}{$\begin{array}{l}\text { Change in intervention group v control group, } \\
\qquad \beta(95 \% \mathrm{CI}), \mathrm{P} \text { value }\end{array}$} \\
\hline & $\mathrm{OM}$ & $6 \mathrm{M}$ & $12 \mathrm{M}$ & OM & $6 \mathrm{M}$ & $12 \mathrm{M}$ & $0-6 \mathrm{M}$ & $0-12 M$ \\
\hline \multicolumn{9}{|l|}{ Physiological fall risk } \\
\hline PPA score & $1.19(0.87)$ & $1.17(0.77)$ & $0.97(0.93)$ & $0.99(0.74)$ & $0.82(0.82)$ & $0.76(0.92)$ & $\begin{array}{l}-0.15(-0.39 \text { to } 0.09) \\
P=0.214\end{array}$ & $\begin{array}{l}-0.01(-0.27 \text { to } 0.26) \\
P=0.955\end{array}$ \\
\hline \multicolumn{9}{|l|}{ Balance, functional mobility, and gait } \\
\hline Standing balance (s; median (IQR)) & $188(69)$ & $189(81)$ & $186(73)$ & $193(95)$ & $209(71)$ & $198(70)$ & 11 (3 to 19$),{ }^{*} \mathrm{P}=\mathrm{NA}$ & 10 (1 to 19$),{ }^{*} \mathrm{P}=\mathrm{NA}$ \\
\hline Maximum lean range AP $(\mathrm{cm})$ & $15(3)$ & $16(4)$ & $18(4)$ & $15(3)$ & $17(4)$ & $19(4)$ & 1 (0 to 2$), P=0.206$ & 1 (0 to 2$), P=0.213$ \\
\hline Coordinated lean (score; median (IQR)) & $7(13)$ & $9(15)$ & $8(11)$ & $7(11)$ & $6(11)$ & $5(12)$ & $-2(-4$ to 0$), P=N A$ & $-1(-3$ to 1$), P=N A$ \\
\hline Timed up-and-go test (s) & $8.6(3.0)$ & $8.7(3.6)$ & $8.6(4.1)$ & $8.5(3.3)$ & $8.5(3.4)$ & $8.2(3.3)$ & $\begin{array}{l}-1.8(-4.4 \text { to } 0.7) \\
P=0.146\end{array}$ & $\begin{array}{l}-1.5(-3.7 \text { to } 0.8) \\
P=0.190\end{array}$ \\
\hline 5 times sit-to-stand test (s) & $12.4(4.3)$ & $12.1(5.2)$ & $11.5(4.7)$ & $12.4(5.4)$ & $12.1(4.6)$ & $11.0(3.8)$ & $\begin{array}{l}0.1(-1.0 \text { to } 1.2) \\
P=0.864\end{array}$ & $\begin{array}{l}-0.4(-1.5 \text { to } 0.6) \\
P=0.411\end{array}$ \\
\hline 10 m walk (s) & $9.0(2.0)$ & $9.1(3.1)$ & $8.8(3.0)$ & $8.9(2.1)$ & $8.7(2.4)$ & $8.6(2.5)$ & $\begin{array}{l}-0.3(-0.8 \text { to } 0.3) \\
P=0.322\end{array}$ & $\begin{array}{l}-0.1(-0.6 \text { to } 0.5) \\
P=0.802\end{array}$ \\
\hline $\begin{array}{l}\text { Short physical performance battery } \\
\text { (score; median (IQR)) }\end{array}$ & $11(2)$ & $11(2)$ & $11(2)$ & $11(2)$ & $11(1)$ & $11(2)$ & $\mathrm{O}(\mathrm{O}$ to 0$), \mathrm{P}=\mathrm{NA}$ & $\mathrm{O}(0$ to 1$), P=N A$ \\
\hline \multicolumn{9}{|l|}{ Stepping performance } \\
\hline Choice stepping reaction time (s) & $1.16(0.20)$ & $1.17(0.17)$ & $1.18(0.23)$ & $1.13(0.18)$ & $1.15(0.19)$ & $1.17(0.18)$ & $\begin{array}{l}0.01(-0.05 \text { to } 0.06) \\
P=0.744\end{array}$ & $\begin{array}{l}0.03(-0.03 \text { to } 0.08) \\
P=0.380\end{array}$ \\
\hline Inhibitory stepping reaction time (s) & $1.32(0.40)$ & $1.32(0.43)$ & $1.32(0.38)$ & $1.26(0.37)$ & $1.36(0.49)$ & $1.29(0.36)$ & $\begin{array}{l}0.10(-0.04 \text { to } 0.23) \\
P=0.143\end{array}$ & $\begin{array}{l}0.03(-0.11 \text { to } 0.17) \\
P=0.645\end{array}$ \\
\hline Stroop stepping reaction time (s) & $1.25(0.42)$ & $1.24(0.39)$ & $1.22(0.39)$ & $1.21(0.34)$ & $1.28(0.38)$ & $1.19(0.34)$ & $\begin{array}{l}0.17(-0.14 \text { to } 0.48) \\
P=0.302\end{array}$ & $\begin{array}{l}0.26(0.12 \text { to } 0.40) \\
P=0.116\end{array}$ \\
\hline \multicolumn{9}{|c|}{ Cognitive performance and executive functions } \\
\hline TMT-A (S; median (IQR)) & $31.9(11.8)$ & $27.5(14.4)$ & $39.6(12.8)$ & $29.8(12.7)$ & $29.7(11.8)$ & $28.4(13.9)$ & $\begin{array}{l}1.8(-0.7 \text { to } 4.2) \\
\mathrm{P}=\mathrm{NA}\end{array}$ & $\begin{array}{l}0.6(-2.0 \text { to } 3.1) \\
\mathrm{P}=\mathrm{NA}\end{array}$ \\
\hline TMT-B (s; median (IQR)) & $85.2(50.7)$ & $90.1(54.3)$ & $84.3(55.8)$ & $87.7(43.2)$ & $87.5(53.5)$ & $87.8(51.8)$ & $\begin{array}{l}1.5(-8.0 \text { to } 11.0) \\
P=N A\end{array}$ & $\begin{array}{l}5.1(-5.1 \text { to } 15.3) \\
P=N A\end{array}$ \\
\hline TMT-B minus TMT-A (s; median (IQR)) & $54.8(44.8)$ & $60.5(44.0)$ & $56.2(44.1)$ & $55.3(36.2)$ & $55.9(38.9)$ & $59.7(41.9)$ & $\begin{array}{l}-0.3(-10.5 \text { to } 9.6) \\
P=N A\end{array}$ & $\begin{array}{l}4.5(-5.8 \text { to } 15.0) \\
P=N A\end{array}$ \\
\hline Victoria Stroop ratio & $2.13(0.87)$ & $1.87(0.92)$ & $1.98(0.91)$ & $1.95(0.74)$ & $1.89(0.89)$ & $1.98(0.96)$ & $\begin{array}{l}0.20(-0.13 \text { to } 0.53) \\
P=0.224\end{array}$ & $\begin{array}{l}0.18(-0.15 \text { to } 0.51) \\
P=0.270\end{array}$ \\
\hline Victoria Stroop errors (median (IQR)) & $4(5)$ & $3(5)$ & $3(4)$ & $3(5)$ & $3(5)$ & $2(4)$ & $\mathrm{O}(0$ to 0$), \mathrm{P}=\mathrm{NA}$ & $\mathrm{O}(0$ to 0$), P=N A$ \\
\hline
\end{tabular}


Adherence to the intervention was higher than reported for previous exercise trials, with $40 \%$ of participants being fully adherent over the first six months and 30\% being fully adherent over the full 24 months compared with pooled estimates of $21 \%$ in previous trials. ${ }^{32}$ These rates are particularly encouraging because adherence was recorded automatically and is therefore a true representation of the actual amount of balance training people received; often adherence rates have lower accuracy because of self-reports or estimates based on number of sessions attended. ${ }^{32}$ Eighty per cent of participants in the intervention group had a median adherence of $105 \mathrm{~min} /$ week over six months, and over half sustained a median adherence of $120 \mathrm{~min} /$ week over 24 months, despite the low level of contact during the study (two home visits in the first month and incidental follow-up calls during the first six months). We acknowledge that when an exercise programme is rolled out to the community with potentially even less follow-up, adherence might be lower. Enjoyment and usability of the StandingTall intervention remained high throughout the study duration. Weekly median exercise durations suggest that the exercises might have become part of the lifestyle of participants who remained in the study. While intentions and selfefficacy towards completing two hours of exercise per week declined over time, this is probably a more realistic reflection of actual long term self-efficacy. The relatively high adherence and zero serious adverse events show promise for upscaling the intervention to a population level.

\section{Strengths and limitations of this study}

The strengths of this study were its large sample size, pragmatic design using a programme that could be delivered as part of routine care, broad inclusion criteria and use of methods designed to reduce the risk of bias such as concealed random allocation to groups, blinded outcome assessment, intention-totreat analyses, and preregistered statistical analysis plan (see assessment in online appendix 4). The primary study limitations were, firstly, the reliance on self-reported falls; however, the weekly e-diaries completed by both groups should have removed any reporting bias. Secondly, similar to many other exercise trials, participant masking was not possible; this might have led to bias by expectation, considering that many outcomes were self-rated. Thirdly, our study design intentionally included more than one outcome measure to account for the many causes of falls, and in theory, the subsequent multiple testing of the results could introduce error. Fourthly, the community dwelling older people who participated in our study were highly educated, had a high percentage of computer ownership, and lived in more affluent areas of Sydney; our results might not generalise to usage in more rural or less affluent areas. Finally, it is possible that our weekly education fact sheets have induced a behaviour change in our control group, reducing our statistical power.

\section{Implications for policy and practice}

New methods for delivery of quality healthcare are required to increase the effectiveness of fall prevention programmes while containing costs and using scarce human resources to maximum effect. The ultimate success of a health promotion programme depends on its effectiveness and its reach and acceptability in the community. A recently published multifactorial fall prevention trial in 5451 older people at high risk of fall injuries showed that all participants had poor balance, and $95 \%$ agreed to take up an exercise programme. ${ }^{38}$ However, the authors indicated that uptake and adherence to community based exercise programmes was low, and the evidence base of these available exercise programmes was uncertain. ${ }^{38}$ StandingTall fills an important gap by helping older people to exercise at home; that is, those who are unable (or unwilling) to attend out-of-house or group exercises, or those who wish to combine group and home based exercises. In their global action plan on physical activity 2018-2030, the World Health Organization advocated exercise as a protective factor in the development of non-communicable diseases such as diabetes, cardiovascular disease, and stroke. ${ }^{39}$ Recent evidence also shows that exercise can delay the onset of dementia and improve mental health in older people. ${ }^{40}$ Also, in light of the covid-19 pandemic, as face-to-face delivery has been curtailed and deconditioning is widespread, e-health can offer an engaging, home based alternative to reduce the long term adverse health effects caused by extended periods of isolation in older people. E-health programmes such as StandingTall can provide older people with an opportunity to stay active, preventing physical deconditioning and concomitant falls, functional dependence, and increased healthcare use while maintaining covid-19 safety recommendations.

\section{Conclusions}

Our results show that a tailored e-health exercise programme is an effective intervention in preventing falls in older people. StandingTall is a scalable intervention and can be easily incorporated into clinical practice, providing healthcare professionals with a platform to remotely set up, monitor, and tailor the programme for their patients. StandingTall offers full user autonomy and requires minimal interaction with healthcare professionals. An economic evaluation is planned to determine whether StandingTall represents value for money.

\section{AUTHOR AFFILIATIONS}

${ }^{1}$ Falls, Balance and Injury Research Centre, Neuroscience Research Australia, Randwick, NSW, Australia

${ }^{2}$ School of Population Health, University of New South Wales, Kensington, NSW, Australia

${ }^{3}$ Faculty of Medicine and Health, School of Public Health, University of Sydney, Sydney, NSW, Australia

${ }^{4}$ Exercise Science Laboratory, School of Kinesiology, Faculty of Medicine, Universidad Finis Terrae, Santiago, Chile

${ }^{5}$ Care and Public Health Research Institute, Department of Health Services Research, Maastricht University, Maastricht, Netherlands ${ }^{6}$ Prince of Wales Hospital Clinical School, University of New South Wales, Kensington, NSW, Australia 
${ }^{7}$ The George Institute for Global Health, University of New South Wales, Newtown, NSW, Australia

${ }^{8}$ College of Medicine and Public Health, Flinders University, Adelaide, SA, Australia

${ }^{9}$ Stats Central, Mark Wainwright Analytical Centre, University of New South Wales, Kensington, NSW, Australia

The authors report that the physiological profile assessment (NeuRA FallScreen) is commercially available through Neuroscience Research Australia.

Contributors: Conceptualisation: KD, SRL, LC, GARZ, JCTC, BT; data collection: KD, TV, AW, JC, GM, LM, KSvS; data analysis: BT, NB, KSvS; data interpretation: KD, TL, KSvS; writing-original draft preparation: KD, KSvS; writing-review and editing: TV, SRL, LC, GARZ, JCTC, TL,

AW, JC, GM, LM, BT, NB; final approval: KD, TV, SRL, LC, GARZ, JCTC, TL, $A W, J C, G M, L M, B T, N B, K S v S$. KD is the guarantor. The corresponding author attests that all listed authors meet authorship criteria and that no others meeting the criteria have been omitted.

Funding: This study was funded by Australian National Health and Medical Research Council grant APP1084739, Gandel Philaptropy, and NeuRA Foundation. KD and SRL were supported by the Australian National Health and Medical Research Council (APP1105106, APP1117171). TL is currently supported by a NHMRC Early Career Fellowship (APP1141392) and National Heart Foundation Postdoctoral Fellowship (award ID 101956). KSvS was supported by a Human Frontier Science Program Fellowship (LT001080/2017). The funders did not have a role in study design; in the collection, analysis, and interpretation of data; in the writing of the report; and in the decision to submit the paper for publication.

Competing interests: All authors have completed the ICMJE uniform disclosure form at www.icmje.org/coi disclosure.pdf and declare: support from the Australian National Health and Medical Research Council, Gandel Philaptropy, NeuRA Foundation, National Heart Foundation, and Human Frontier Science Program for the submitted work; no financial relationships with any organisations that might have an interest in the submitted work in the previous three years; no other relationships or activities that could appear to have influenced the submitted work.

Ethical approval: The trial was approved by the University of New South Wales ethics committee in December 2014 (HC\#14/266).

Data sharing: Deidentified participant data may be accessed by researchers who provide a methodologically sound proposal. Proposals should be directed to KD (k.delbaere@neura.edu.au) and data are available from the date of publication of this article. The study protocol is available as a free access publication ${ }^{8}$ and the statistical analysis plan is available on OpenScience framework (https://osf.io/42gje/).

The corresponding author (KD) affirms that the manuscript is an honest, accurate, and transparent account of the study being reported; that no important aspects of the study have been omitted; and that any discrepancies from the study as originally planned (and, if relevant, registered) have been explained.

Dissemination to participants and related patient and public communities: Outcomes will be disseminated through study newsletters, community events, social media, and media releases.

Provenance and peer review: Provenance and peer review: Not commissioned; externally peer reviewed.

This is an Open Access article distributed in accordance with the Creative Commons Attribution Non Commercial (CC BY-NC 4.0) license, which permits others to distribute, remix, adapt, build upon this work non-commercially, and license their derivative works on different terms, provided the original work is properly cited and the use is noncommercial. See: http://creativecommons.org/licenses/by-nc/4.0/.

1 James SL, Lucchesi LR, Bisignano C, Castle CD, Dingels ZV, Fox JT, et al. The global burden of falls: global, regional and national estimates of morbidity and mortality from the Global Burden of Disease Study 2017. Inj Prev . 2020;26:i3-i11

2 Sherrington C, Fairhall NJ, Wallbank GK, Tiedemann A, Michaleff ZA, Howard K, et al. Exercise for preventing falls in older people living in the community. Cochrane Database of Systematic Reviews. 2019.

3 Nyman SR, Victor CR. Older people's participation in and engagement with falls prevention interventions in community settings: an augment to the Cochrane systematic review. Age Ageing 2012;41:16-23. doi:10.1093/ageing/afr103

4 Simek EM, McPhate L, Haines TP. Adherence to and efficacy of home exercise programs to prevent falls: a systematic review and meta-analysis of the impact of exercise program characteristics. Prev Med 2012;55:262-75. doi:10.1016/j.ypmed.2012.07.007

5 Valenzuela T, Okubo Y, Woodbury A, Lord SR, Delbaere K. Adherence to technology-based exercise programs in older adults: a systematic review. J Geriatr Phys Ther 2018;41:49-61.

6 McGarrigle L, Boulton E, Todd C. Map the Apps: a rapid review of digital approachesto support the engagement of older adults instrength and balance exercises. Physical Medicine and Rehabilitation.2020. "https://doi.org/" doi:10.21203/ rs.3.rs-63743/v1

7 Lamb SE, Jørstad-Stein EC, Hauer K, Becker C, Prevention of Falls Network Europe and Outcomes Consensus Group. Development of a common outcome data set for fall injury prevention trials: the Prevention of Falls Network Europe consensus. J Am Geriatr Soc 2005;53:1618-22. doi:10.1111/j.1532-5415.2005.53455.x

8 Delbaere K, Valenzuela T, Woodbury A, et al. Evaluating the effectiveness of a home-based exercise programme delivered through a tablet computer for preventing falls in older communitydwelling people over 2 years: study protocol for the Standing Tall randomised controlled trial. BMJ Open 2015;5:e009173. doi:10.1136/bmjopen-2015-009173

9 Pfeiffer E. A short portable mental status questionnaire for the assessment of organic brain deficit in elderly patients. J Am Geriatr Soc 1975;23:433-41. doi:10.1111/j.1532-5415.1975.tb00927.x

10 Lord SR, Menz HB, Tiedemann A. A physiological profile approach to falls risk assessment and prevention. Phys Ther 2003;83:237-52. doi:10.1093/ptj/83.3.237

11 Brandler TC, Oh-Park M, Wang C, Holtzer R, Verghese J. Walking while talking: investigation of alternate forms. Gait Posture 2012;35:1646. doi:10.1016/j.gaitpost.2011.08.003

12 Csuka M, McCarty DJ. Simple method for measurement of lower extremity muscle strength. Am J Med 1985;78:77-81. doi:10.1016/0002-9343(85)90465-6

13 Podsiadlo D, Richardson S. The timed “Up \& Go": a test of basic functional mobility for frail elderly persons. J Am Geriatr Soc 1991;39:142-8. doi:10.1111/j.1532-5415.1991.tb01616.x

14 Guralnik JM, Simonsick EM, Ferrucci L, et al. A short physical performance battery assessing lower extremity function: association with self-reported disability and prediction of mortality and nursing home admission. / Gerontol 1994:49:M85-94. doi:10.1093/ geronj/49.2.M85

15 Bohannon RW. Comfortable and maximum walking speed of adults aged 20-79 years: reference values and determinants. Age Ageing 1997;26:15-9. doi:10.1093/ageing/26.1.15

16 Schoene D, Smith ST, Davies TA, Delbaere K, Lord SR. A Stroop Stepping Test (SST) using low-cost computer game technology discriminates between older fallers and non-fallers. Age Ageing 2014:43:285-9. doi:10.1093/ageing/aft157

17 Schoene D, Delbaere K, Lord SR. Impaired response selection during stepping predicts falls in older people-a cohort study. J Am Med Dir Assoc 2017;18:719-25. doi:10.1016/j.jamda.2017.03.010

18 Nasreddine ZS, Phillips NA, Bédirian V, et al. The Montreal Cognitive Assessment, MoCA: a brief screening tool for mild cognitive impairment. J Am Geriatr Soc 2005;53:695-9. doi:10.1111/j.15325415.2005.53221.x

19 Reitan RM, Wolfson D. The Halstead-Reitan neuropsychological test battery: Theory and clinical interpretation. Neuropsychology Press, 1985.

20 Trenerry MR, Crosson B, DeBoe J, Leber W. Stroop neuropsychological screening test. Psychological Assessment Resources, 1989.

21 Delbaere K, Smith ST, Lord SR. Development and initial validation of the iconographical galls efficacy scale. J Gerontol A 2011;66A:67480 .

22 Kroenke K, Spitzer RL, Williams JB. The PHQ-9: validity of a brief depression severity measure. J Gen Intern Med 2001;16:606-13. doi:10.1046/j.1525-1497.2001.016009606.x

23 Gatt JM, Burton KL, Schofield PR, Bryant RA, Williams LM. The heritability of mental health and wellbeing defined using COMPAS-W, a new composite measure of wellbeing. Psychiatry Res 2014:219:204-13. doi:10.1016/i.psychres.2014.04.033

24 Üstün TB, Chatterji S, Kostanjsek N, et al, WHO/NIH Joint Project. Developing the World Health Organization disability assessment schedule 2.0. Bull World Health Organ 2010;88:815-23. doi:10.2471/BLT.09.067231

25 Viney R, Norman R, King MT, et al. Time trade-off derived EQ-5D weights for Australia. Value Health 2011;14:928-36. doi:10.1016/i. jval.2011.04.009

26 Richardson JR, Peacock SJ, Hawthorne G, lezzi A, Elsworth G, Day NA. Construction of the descriptive system for the Assessment of Quality of Life AQoL-6D utility instrument. Health Qual Life Outcomes 2012;10:38. doi:10.1186/1477-7525-10-38

27 Delbaere K, Hauer K, Lord SR. Evaluation of the incidental and planned activity questionnaire (IPEQ) for older people. Br J Sports Med 2010;44:1029-34. doi:10.1136/bjsm.2009.060350 
28 van Schooten KS, Rispens SM, Elders PJ, Lips P, van Dieën JH, Pijnappels M. Assessing physical activity in older adults: required days of trunk accelerometer measurements for reliable estimation. J Aging Phys Act 2015;23:9-17. doi:10.1123/JAPA.2013-0103

29 Bangor A, Kortum P, Miller J. Determining what individual SUS scores mean: Adding an adjective rating scale. J Usability Stud 2009;4: 114-23.

30 Kendzierski D, DeCarlo KJ. Physical activity enjoyment scale: two validation studies. / Sport Exerc Psychol 1991;13:50-64. doi:10.1123/jsep.13.1.50

31 Everett B, Salamonson Y, Davidson PM. Bandura's exercise selfefficacy scale: validation in an Australian cardiac rehabilitation setting. Int J Nurs Stud 2009;46:824-9. doi:10.1016/j. ijnurstu.2009.01.016

32 Yardley L, Donovan-Hall M, Francis K, Todd C. Attitudes and beliefs that predict older people's intention to undertake strength and balance training. J Gerontol B Psychol Sci Soc Sci 2007;62:119-25. doi:10.1093/geronb/62.2.P119

33 Huque MH, Carlin JB, Simpson IA, Lee KJ. A comparison of multiple imputation methods for missing data in longitudinal studies. BMC Med Res Methodol 2018;18:168. doi:10.1186/s12874-018-0615-6

34 Finnegan S, Seers K, Bruce J. Long-term follow-up of exercise interventions aimed at preventing falls in older people living in the community: a systematic review and meta-analysis. Physiotherapy 2019;105:187-99. doi:10.1016/j.physio.2018.09.002

35 Zhao R, Bu W, Chen X. The efficacy and safety of exercise for prevention of fall-related injuries in older people with different health conditions, and differing intervention protocols: a metaanalysis of randomized controlled trials. BMC Geriatr 2019;19:341. doi:10.1186/s12877-019-1359-9

36 Howe TE, Rochester L, Neil F, Skelton DA, Ballinger C. Exercise for improving balance in older people. Cochrane Database Syst Rev 2011;(11):CD004963. doi:10.1002/14651858.CD004963. pub3

37 McClure NS, Sayah FA, Xie F, Luo N, Johnson JA. Instrument-defined estimates of the minimally important difference for EQ-5D-5L index scores. Value Health 2017;20:644-50. doi:10.1016/j. jval.2016.11.015

38 Bhasin S, Gill TM, Reuben DB, et al, STRIDE Trial Investigators. A randomized trial of a multifactorial strategy to prevent serious fall injuries. N Engl J Med 2020;383:129-40. doi:10.1056/ NEJMoa2002183

39 Global action plan on physical activity 2018-2030: more active people for a healthier world. World Health Organization. 2018 https://www.who.int/ncds/prevention/physical-activity/globalaction-plan-2018-2030/en/

40 Langhammer B, Bergland A, Rydwik E. The importance of physical activity exercise among older people. Biomed Res Int 2018:2018:7856823. doi:10.1155/2018/7856823

Supplementary information: online supplementary material 